\title{
Der scheinbare Autonomieraub am Lebemann und wie mich sein Lächeln eines Besseren belehrte
}

\author{
Nathan Elia Schmid \\ Medizinstudent im ersten Jahr, Universität Zürich
}

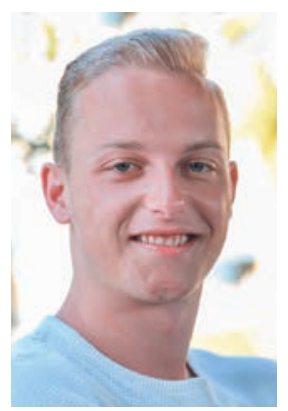

Umgeben von unberührter Natur und der Ruhe des idyllischen Kurortes inmitten des Sarganserlandes schlängelt sich der Rhein durch das Tal. Ein sonniger und wolkenfreier Himmel überstrahlt die Szene an diesem Tag, als möchte er jemandem die letzte Ehre erweisen. Die Urne meines Grossvaters in der Hand, taste ich mich Schritt für Schritt ins seichte Flussbett. Mit zittrigen Händen und Tränen in den Augen nehme ich Abschied von einem Stück seliger Kindheit. Hinter uns liegen Jahre der Hilflosigkeit und Trauer beim Begleiten des Grossvaters in die Demenz. Mit jeder Streubewegung vermischen sich die trüben Gedanken mit dem klaren Wasser und machen sich zusammen mit der Asche meines Grossvaters auf die Reise flussabwärts. Der lange Leidensweg meines geliebten Grossvaters hat mich in den letzten Jahren emotional stark beschäftigt. Den Weg vom vitalitätssprühenden Bonvivant zum geistig umnachteten Schatten seiner selbst habe ich in meiner Jugendzeit miterlebt. Man fragt sich nun vielleicht, was das Ganze mit Ethik zu tun hat. Wir als Familie hatten beschlossen, meinen Grossvater, nachdem ihn seine körperlich gebrechliche Ehefrau über zehn Jahren zu Hause gepflegt hatte, in einem Pflegeheim für Demenzkranke unterzubringen. Dies geschah - wie mir damals schien - gegen seinen über die Jahre stets unmissverständlich geäusserten Willen und entgegen seinem Naturell. Vielleicht wird nun klarer, inwiefern ein demenzkrankes Familienmitglied einen ethischen Konflikt auslösen kann. Wie jedoch kam es dazu, dass wir uns in einer solch heiklen Situation wiederfanden?

Mein Grossvater war ein Lebemann. Als passionierter Landjäger und späterer Generalsekretär des Schweizerischen Polizeimeisterverbandes lebte er mit meiner Grossmutter und fünf gemeinsamen Kindern in den Bündner Bergen. Neben seiner Berufung als Gesetzesund Ordnungshüter war er leidenschaftlicher Berggänger und Naturfreund, welcher mit Hut und Spazierstock auch im Alter noch so manchen Berg zu erklimmen vermochte. Er liebte die gute Gesellschaft

und konnte mit seinem Schalk und seiner Lebensfreude ein einfaches Abendessen in ein Fest verwandeln. Da war aber auch seine Rolle als Patriarch. Er, das alles bestimmende und von niemandem offen in Frage gestellte Familienoberhaupt. Was er sagte, galt, und was er nicht wollte - das liess man besser bleiben. Im Alter von 69 Jahren erlitt er - aus scheinbar voller Gesundheit heraus - einen Hirnschlag, der sein Sprachzentrum und somit sein Sprechvermögen und das Sprachverstehen in Mitleidenschaft zog. Nach kurzem Aufenthalt in einer Rehabilitationsklinik wurde der zumindest körperlich rüstig gebliebene Rentner nach Hause entlassen, wo ihn seine Frau die nächsten zehn Jahre seines Lebens liebevoll betreuen und aufopferungsvoll pflegen sollte. Die zunehmende und am Schluss schwere Demenz veranlasste uns als Familie, uns für eine Verlegung in eine speziell für Demenzkranke eingerichtete Institution zu entscheiden. Unversehens fand er sich eines Tages, fernab seines vertrauten Umfeldes, in einem Alters- und Pflegeheim wieder. Doch was geschah dazwischen? Was bewog uns zu diesem Entscheid und welche Emotionen, welche Argumente spielten eine tragende Rolle?

Aus der Sicht meines Grossvaters, welcher immer strikt gegen einen Umzug jeglicher Art aus seinen eigenen vier Wänden war, musste sich dieser Entscheid wie ein Raub seiner Autonomie angefühlt haben. Er wollte nicht weg - wie konnte man sich ihm nur widersetzen? Ein zentraler Aspekt des ordnungsgemässen und ethisch korrekten Entscheidungsfindungsprozesses ist doch das Miteinbeziehen des Patienten. Unabhängig vom Grad seiner Urteilsfähigkeit steht dem Patienten ein Recht zu, Teil der Verhandlungen und Diskussionen zu sein. Kann er auf verbaler Ebene nicht mehr ausreichend kommunizieren, was bei einer gemischten Sprachstörung zweifellos der Fall ist, müssen die Aspekte der non- und paraverbalen Kommunikation berücksichtigt werden. Dadurch hat der Demenzkranke auch ohne Worte die Möglichkeit, sein Wohlbefinden oder seine Missstimmung kundzutun. Retro- 
spektiv haben wir das viel zu wenig berücksichtigt. Dies lässt wenige Zweifel daran, dass der Entscheid aus der Sicht meines Grossvaters ethisch unzureichend aufgearbeitet wurde.

Aus der Sicht meiner Grossmutter musste die Situation zermürbend gewesen sein. Für sie, welche erst jahrelang in einer subalternen Position verharrt hatte und ohne Vorwarnung plötzlich die gesamte Verantwortung tragen musste, konnte sich das Vorhaben einer Verlegung nur falsch angefühlt haben. Meiner Grossmutter war es trotz der zunehmenden zeitlichen und örtlichen Desorientierung ihres Ehemanns gelungen, ein personenzentriertes Arrangement seines Lebensumfeldes zu schaffen, um ihm das Wohnen zu Hause weitestgehend zu erleichtern: Die Vorratskammer wurde fortan vom Keller in die Badewanne verlegt; Zwiebeln, Kartoffeln und Milchkartons liebevoll nebeneinander postiert. Auf dem Esstisch stellte sie alles immer exakt an denselben Platz, damit er seinen gewohnten Aufgaben wie Kaffeeeingiessen oder Brotschneiden trotz der durch den Hirnschlag verursachten Gesichtsfeldeinschränkung weiterhin nachkommen konnte. Sie verstand es, in seiner Welt mitzuleben und seiner eigenen Realität Glauben zu schenken, um ihm Sicherheit und Achtung zu vermitteln und ihm so zumindest einen kleinen Anteil am gewohnten Leben zu erhalten. Somit sorgte sie für eine optimale Betreuungs- und Behandlungsqualität. Nichtsdestotrotz musste sie sich eingestehen, dass sie die Verantwortung nicht länger tragen konnte. Die Desorientierung ihres Ehemanns, der komplette Verlust des Zeitgefühls, somit auch des Tag-Nacht-Rhythmus und seine immer häufiger wiederkehrenden unkontrollierten Aggressionsausbrüche gegen sie liessen ein sicheres Leben zu Hause nicht länger zu. Somit entstand für sie ein Konflikt zwischen dem eigenen Zerbrechen an der Situation und der Befürchtung, im Falle eines Wegtransportes ihres Mannes dem Prinzip der Care-Ethik nicht länger gerecht werden zu können.

Als Medizinstudent im ersten Studienjahr empfinde ich die Beurteilung der Situation als wesentlich angenehmer. Da war ein körperlich rüstiger Patient fortgeschrittenen Alters, welcher nach einem Hirnschlag über die Jahre hinweg eine schwere Demenz entwickelt hatte. An eine Pflege zu Hause durch seine Ehefrau, welche selbst unter schweren körperlichen Gebrechen litt und den alltäglichen Pflichten nur noch mühselig nachkam, war nicht länger zu denken. Weiter kam dazu, dass der Patient aufgrund seiner Überforderung mit den Problemen des Alltags, seiner schweren Orientierungslosigkeit und seines impulsiven Verhaltens sich und seine Frau zunehmend gefährdete. Ein professionelles Setting in einer Anstalt für demenzerkrankte Personen mit vollumfänglicher Betreuung schien die einzige in Betracht zu ziehende Option darzustellen. So weit, so gut, nur: Wie stehe ich als Enkel zu diesem Entscheid?

Ich bin zwiegespalten, ob wir als Familie richtig gehandelt und unseren «Tat» - das Bündner Wort für Grossvater - mit unserem Beschluss zur Verlegung nicht im Stich gelassen haben. Im Wissen, dass dieser Entscheid eine paternalistische Handlung darstellte und wir über den Kopf unseres Grossvaters hinweg entschieden, ihm scheinbar die Autonomie raubten; sind wir aus ethischer Sicht nun gescheitert oder gibt es etwas, das ich bisher nicht berücksichtigt habe etwas, was meine Zweifel abschwächen würde? Sein Lächeln vielleicht, an welchem ich bis tief in die Demenz hinein den Lebemann erkennen konnte? Die grenzenlose Freude in seinen Augen, die Tränen der Dankbarkeit und der emotionalen Überforderung, als wir ihn jeweils auf der Demenzstation besuchten; wenn ich diese Aspekte der nonverbalen Kommunikation, welche wir im Entscheidungsprozess vernachlässigt haben, nun auf die Weise berücksichtigen kann hilft mir das, meinen Zwiespalt zu beseitigen?

Ich finde ja. In meinen Augen sprechen diese Emotionen eine überzeugende und, obwohl wortlos, nicht minder deutliche Sprache. Sie bestärkt mich heute, dass die Verlegung weg von zu Hause und der dadurch weiter begünstigten Desorientierung ethisch durchaus sorgfältig bedachte Aspekte aufweist. Es war sein Lächeln, das mich eines Besseren belehrte.

Als unvoreingenommener Medizinstudent und gleichzeitig persönlich geprägter Enkel bleibt mir somit ein Gefühl der Faszination gepaart mit Ehrfurcht. Vielleicht bietet sich mir als praktizierendem Arzt auch einmal die Möglichkeit, mich mit einem solchen Fall auseinandersetzen zu dürfen. Was subjektiv aus medizinischer Sicht auf den ersten Blick vielleicht klar erscheint, entpuppt sich aufgrund familien- und patientenbezogener Konflikte als ethisch komplexe Angelegenheit. Ich erhoffe mir, nach bestem Wissen und Gewissen zu handeln und letztendlich zum Wohle der Patientin oder des Patienten zu entscheiden - bis dahin allerdings wird noch viel Wasser den Rhein hinunterfliessen. 